\title{
Enfermedad de Chagas en el sistema nervioso central en paciente con infección por VIH: dificultades diagnósticas y terapéuticas
}

\author{
Federico Simioli, Milagro Sánchez-Cunto, Elsa Velázquez, Susana Lloveras y Tomás Orduna
}

Hospital de Enfermedades Infecciosas Francisco Javier Muñiz, Buenos Aires, Argentina. (FS, MSC, SLI, TO) Instituto Nacional de Parasitología Dr. Mario Fatala Chaben, Buenos Aires, Argentina (EV).

Recibido: 22 de diciembre de 2015 Aceptado: 4 de noviembre de 2016

Correspondencia a: Federico Simiol fedesimioli@hotmail.com

\section{Chagas disease in the central nervous system in patient infected with HIV: diagnostic and therapeutic difficulties}

Chagas disease (ChD), caused by the protozoan Trypanosoma cruzi, is an endemic anthropozoonosis in Latin America, linked to deficients socio-economic and cultural aspects and is considered one of the neglected tropical diseases. We report a fatal case of Chagas disease reactivation with central nervous system involvement in a patient with HIV infection, whose diagnosis was confirmed by positive PCR (polymerase chain reaction) test of blood, with treatment response efficiency with benznidazol and management and etiologic treatment was difficult due to limited number of antitrypanosomal drugs and the occurrence of frequent and serious adverse effects.

Key words: Chagas, reactivation, HIV, PCR.

Palabras clave: Chagas, reactivación, VIH, RPC.

\section{Introducción}

$\mathrm{L}$ a enfermedad de Chagas o tripanosomiasis americana es una antropo-zoonosis endémica en Latinoamérica vinculada con aspectos socioeconómico-culturales deficitarios, como viviendas de tipo rancho, y considerada una de las enfermedades desatendidas. Es causada por el protozoo flagelado Trypanosoma cruzi, -descubierto en 1909 en Minas Gerais, Brasil, por Carlos Chagas-, quien, en homenaje a su maestro Osvaldo Cruz, le dio la citada denominación ${ }^{1}$. Es una patología endémica en 21 países de las Américas, aunque producto de las migraciones de personas infectadas constituye un problema de salud para países no endémicos de todo el mundo. El principal mecanismo de transmisión es vectorial, por hemípteros de la subfamilia Triatominae (con alimentación hematófaga). Infectan personas expuestas a su picadura, al depositar sus heces con parásitos que ingresan por piel o mucosas. Otras modalidades de transmisión son: transfusional, congénita o vertical, secundaria a trasplantes de órganos, adicción a drogas intravenosas (por el hábito de compartir agujas) ${ }^{2}$, oral $^{3} \mathrm{y}$ accidentes de laboratorio ${ }^{4}$. Se estima que en la región endémica, cerca de 100 millones de personas están en riesgo de infectarse y ocho millones están infectadas. Se producen anualmente 56.000 nuevos casos por todas las formas de transmisión, y 12.000 muertes aproximadamente ${ }^{5}$. La reactivación de la enfermedad de Chagas crónica es infrecuente; sin embargo, puede producirse en pacientes inmunocomprometidos graves, especialmente en aquellos con compromiso de la inmunidad celular, como en el caso de la infección por VIH/SIDA. El mayor riesgo de reactivación se presenta cuando el recuento de linfocitos T CD4+ cae por debajo de 100 céls $/ \mu \mathrm{L}^{3}$. Estas reactivaciones suelen acompañarse de parasitemias elevadas, y afectan principalmente al sistema nervioso central (SNC) y menos comúnmente al miocardio. En el SNC, la forma clínica de presentación más frecuente es como una masa ocupante, pseudotumor o "chagoma", indistinguible clínica y radiológicamente de la toxoplasmosis cerebral y de otras encefalitis necrosantes. Otra forma de presentación, menos frecuente, es la meningoencefalitis difusa, que suele coincidir con la observación de abundantes tripomastigotes en el líquido cefalorraquídeo $(\mathrm{LCR})^{6-12}$. Estas formas clínicas graves, en ocasiones, son difíciles de tratar por la escasez de opciones terapéuticas y por la toxicidad de los fármacos disponibles.

\section{Caso clínico}

Mujer de 36 años, oriunda de Los Ralos, Tucumán, provincia del noroeste de la República Argentina, con antecedentes de infección por VIH desde el año 2002, sin terapia antiretroviral (TARV) ni controles por su enfermedad, hepatitis $\mathrm{C}$ y enfermedad de Chagas crónica, este último diagnóstico realizado mediante tres pruebas serológicas positivas (ELISA, hemaglutinación indirecta (HAI) e inmunofluorescencia (IFI). Ingresó al Hospital de Enfermedades Infecciosas Francisco J. Muñiz de la 
Ciudad de Buenos Aires por un síndrome convulsivo agudo. En el examen físico presentaba una paresia braquio-crural izquierda y facial central derecha, ataxia, dismetría y nistagmus.

Entre los exámenes de laboratorio al ingreso destacaba un hemograma con hematocrito 40,2\%, hemoglobina $13,5 \mathrm{~g} / \mathrm{dl}$, leucocitos $3.200 / \mathrm{mm}^{3}$, plaquetas $155.000 / \mathrm{mm}^{3}$, VHS $40 \mathrm{~mm} / \mathrm{h}$, GOT $187 \mathrm{U} / \mathrm{l}$, GPT $97 \mathrm{U} / \mathrm{l}$, exámenes de coagulación normales, VDRL, HBsAg y antiHBc no reactivos, carga viral para VHC 14.279 .000 copias $(7,16$ log). El recuento de LT CD4+ era de 79 céls $/ \mu \mathrm{L}^{3}(8 \%)$. Se realizaron radiografía de tórax, ECG y ecocardiograma que fueron normales.

En la RM de cerebro con contraste se observó una extensa área de señal patológica hiperintensa en FLAIR, T2 y difusión con discreto efecto de masa y algunos sectores de refuerzo heterogéneo luego de inyectado el contraste en proyección córtico-subcortical que ingresaba a los pedúnculos cerebelosos del lado derecho y con extensión al tronco cerebral e imágenes de similar comportamiento de señal aunque sin efecto de masa ni refuerzo con el contraste en la proyección córtico-subcortical frontal lateral a predominio izquierdo (Figura 1). Se realizó una punción lumbar con presión de apertura de $12 \mathrm{~cm} \mathrm{H}_{2} \mathrm{O}$, obteniéndose un LCR límpido, incoloro, proteínas 0,47 $\mathrm{mg} / \mathrm{dl}$, glucosa $78 \mathrm{mg} / \mathrm{dl}, 11$ céls $/ \mathrm{mm}^{3}$ (100\% mononucleares) con un examen directo y cultivo bacteriológico, micológico y para micobacterias negativos. El estudio molecular (RPC) para VHS-1 y 2, VVZ, CMV, VHH-6 y VEB fue negativo. Se realizó un microhematocrito de Strout en dos oportunidades, sin observación de parásitos.

La serología para toxoplasmosis fue positiva (IgG positiva 1/64 e IgM negativa por IFI), por lo que inició tratamiento empírico como una toxoplasmosis cerebral con pirimetamina y sulfadiazina en dosis habituales.

La paciente presentó progresión clínica del síndrome cerebeloso luego de dos semanas de tratamiento, por lo que se agregó tratamiento con benznidazol $300 \mathrm{mg}$ al día por presumir una probable reactivación de una infección chagásica. Se realizó una RPC para Chagas en plasma y en LCR, siendo positiva la muestra plasmática (Figura 2).

La paciente evolucionó con una mejoría del cuadro clínico y de las lesiones observadas en las imágenes. Se realizó una RM cerebral con contraste a los 35 días de iniciado el tratamiento antichagásico, en la que se evidenció una reducción significativa de las lesiones, con la que se decidió el alta hospitalaria y seguimiento ambulatorio. A los 15 días del tratamiento con benznidazol presentó una plaquetopenia y aumento de las transaminasas hepáticas por lo que continuó tratamiento con nifurtimox $240 \mathrm{mg}$ al día. A los 11 días de este último tratamiento presentó neuropatía periférica por lo que se reinstauró benznidazol. La paciente no cumplió con los controles clínicos, y una semana después de iniciar nuevamente el tratamiento con

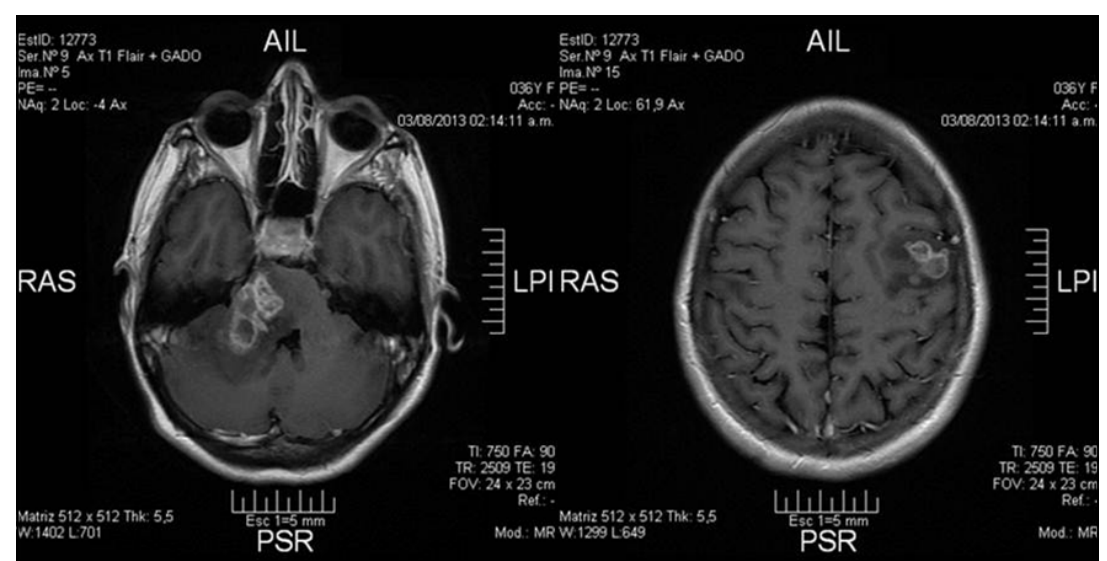

Figura 1. RM axial T1 Flair con gadolinio: formaciones nodulares heterogéneas que se refuerzan con el contraste intravenoso en fosa posterior derecha y lóbulo frontal lateral izquierdo.

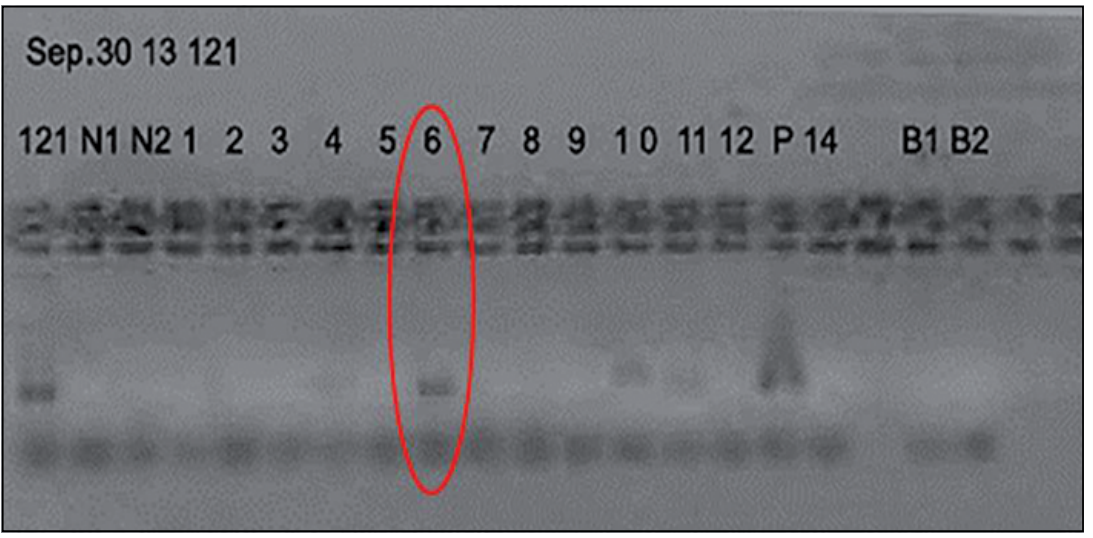

Figura 2. Amplificación del ADN extraído de LCR con primers 121/122 de 330 pb en 2\% de gel de agarosa teñido con bromuro de etidio. La extracción de ADN fue realizada con un kit comercial (High Pure PCR Template preparation kit, Roche Applied Science) de acuerdo con protocolo del fabricante. Calle 9: amplificación del ADN extraído de LCR con primers 121/122 (6); Calle 1: control de banda (121); calle 2 y 3 : controles negativos (N1 y N2); calle 16: control positivo (P); calle 19 y 20: Blancos (B1 y B2).

benznidazol fue internada por fiebre y pancitopenia. Presentó un cuadro clínico de falla multiorgánica de presunta etiología séptica y falleció a las 24 horas del ingreso.

\section{Discusión}

Las formas graves de la enfermedad de Chagas en pacientes con infección por VIH se producen generalmente por reactivación de una infección crónica previa, hasta ese momento oligosintomática o asintomática. Las formas de presentación más frecuentes son: el compromiso de SNC como masa cerebral o meningoencefalitis difusa aguda $^{6,13,14}$ y miocarditis ${ }^{15}$, ambas con elevada mortalidad. Otras manifestaciones menos frecuentes son la peritonitis 
espontánea $^{16}$ con identificación de tripomastigotes de $T$. cruzi en el líquido ascítico y la diarrea crónica con identificación de amastigotes de $T$. cruzi en biopsias duodenales ${ }^{17}$.

Las lesiones cerebrales en la enfermedad de Chagas son clínica y radiológicamente indistinguibles de otros procesos neurológicos que afectan a pacientes con SIDA ${ }^{13}$. El primer diagnóstico diferencial es la toxoplasmosis cerebral, aunque deben considerarse otros como la leucoencefalopatía multifocal progresiva, tuberculomas, criptococomas, nocardiosis, abscesos piógenos y linfoma primario del $\mathrm{SNC}^{15}$. Para obtener un diagnóstico de certeza debe realizarse el estudio histopatológico de muestras obtenidas por biopsia de cerebro. La biopsia cerebral en pacientes con infección por VIH/SIDA y masa ocupante cerebral debe realizarse entre los 10 y 15 días luego de iniciado el tratamiento antitoxoplásmico y de no obtenerse la respuesta al mismo. Por esta razón, en pacientes con infección por VIH/SIDA y lesiones neurológicas focales que vivan o provengan de zonas endémicas debe descartarse enfermedad de Chagas. Debe realizarse serología para Chagas y búsqueda de parásitos en sangre, a través del microhematocrito, la gota gruesa o el método de triple centrifugación de Strout, métodos de bajo costo que permiten un resultado precoz, certeza diagnóstica y sencillez operativa; sin embargo, su sensibilidad es operador dependiente. Cabe recalcar que los estudios serológicos pueden ser negativos, ya que los pacientes pueden perder los anticuerpos específicos en estadios avanzados del SIDA.

En pacientes con meningoencefalitis por Chagas el examen del LCR suele presentar una pleocitosis discreta $\left(<100\right.$ céls $/ \mathrm{mm}^{3}$ con predominio de linfocitos mononucleares), hiperproteinorraquia leve o moderada y glucorraquia usualmente normal (ocasionalmente baja), indistinguible de otras patologías oportunistas que afectan el SNC en pacientes con infección por VIH. Debe destacarse que en el caso de la enfermedad de Chagas, el parásito habitualmente está presente en el LCR, pudiendo observarse en forma de tripomastigotes en el examen en fresco o con la coloración de Giemsa ${ }^{6}$.

Las pruebas actuales para el diagnóstico de la invasión del SNC en la enfermedad de Chagas tienen una baja sensibilidad y, en algunas situaciones, no permiten establecer la etiología. La biología molecular, mediante la técnica de RPC, es una herramienta que permite amplificar cantidades mínimas de ADN del parásito con alta especificidad y sensibilidad. Aunque no es una prueba de rutina, puede ser útil en el diagnóstico de casos en los cuales la serología es dudosa y la parasitemia o parasitorraquia son negativas ${ }^{18,19}$. Existen investigaciones sobre la aplicabilidad de técnicas moleculares para detectar $T$. cruzi en sangre y LCR para la caracterización genética de la población parasitaria asociada con la invasión del $\mathrm{SNC}$ en los seres humanos ${ }^{20}$.
Desde el punto de vista terapéutico sólo se cuenta con dos fármacos antiparasitarios, nifurtimox y benznidazol, ambas con potenciales efectos colaterales y adversos graves, por lo cual los pacientes deben tener un estricto seguimiento médico, para el reconocimiento y tratamiento precoz de los mismos y/o suspensión del fármaco en uso. Los eventos adversos del tratamiento tripanocida son variables según el medicamento utilizado. En general, la mayoría se presenta entre los 15 y 30 días de iniciada la administración del fármaco. Se debe recordar que la frecuencia e intensidad es mayor a medida que aumenta la edad de los pacientes.

Entre los eventos adversos observados con el tratamiento tripanocida pueden mencionarse ${ }^{21}$ : dermatológi$\cos$, angioedema ${ }^{22}$, hematológicos, neuropatía periférica (con benznidazol); toxicidad en SNC (con nifurtimox); gastrointestinales y hepatotoxicidad (con ambos). Erupción cutánea, síntomas gastrointestinales y trastornos del sistema nervioso son las razones más comunes para las interrupciones de tratamiento con estos fármacos ${ }^{23}$. En ocasiones, en función de la gravedad del cuadro clínico, puede ser necesario re-exponer al paciente a un medicamento que ha provocado efectos adversos, lo que puede generar nuevas toxicidades ${ }^{21}$. En nuestro caso clínico, la paciente presentó varios de los efectos adversos descritos como plaquetopenia y aumento de transaminasas con el uso de benznidazol y neuropatía periférica con el uso de nifurtimox. Esto demuestra que no existe un tratamiento etiológico eficaz sin efectos colaterales y que se necesitan nuevos fármacos eficaces con menos efectos secundarios. Lamentablemente la industria farmacéutica internacional ha demostrado escaso interés en esta parasitosis que pertenece a las enfermedades olvidadas o dejadas de lado ("neglected diseases") y que afectan a una población de escasos recursos ${ }^{24}$.

El pronóstico y la supervivencia de estos pacientes es desfavorable, aun con tratamiento adecuado. Sin embargo, la terapia antiretroviral de alta eficacia (HAART, Highly Active Antiretroviral Therapy) permitió mejorar los recuentos de linfocitos T CD4 y el pronóstico de los pacientes con VIH/SIDA. El tratamiento precoz de la encefalitis chagásica en pacientes con SIDA ha logrado mejorar la supervivencia; por esta razón, en algunos casos, debe considerarse el tratamiento empírico con benznidazol en pacientes con lesiones graves del SNC de etiología no definida ${ }^{25}$. Tanto con benznidazol como con nifurtimox, la duración recomendada del tratamiento es de 60 días $^{21}$. La profilaxis secundaria a largo plazo con benznidazol debe indicarse en todos aquellos pacientes con VIH/SIDA que superen un episodio agudo. En el año 2000, la OPS recomendó el uso de benznidazol en dosis de $5 \mathrm{mg} / \mathrm{kg} /$ día, tres veces a la semana, por tiempo indeterminado. Nifurtimox puede usarse con el mismo propósito $^{26}$. 


\section{Conclusión}

En Latinoamérica y en países con migrantes provenientes de áreas endémicas para enfermedad de Chagas, ésta patología debe incluirse dentro de los diagnósticos diferenciales de una masa ocupante cerebral, asociada o no a otros focos de reactivación, en pacientes con VIH/SIDA.

El diagnóstico de la reactivación de la enfermedad de Chagas en pacientes con infección por VIH/SIDA debe ser precoz, ya que un tratamiento adecuado mejora el pronóstico y la supervivencia de estos pacientes. Las pruebas de biología molecular como la RPC en plasma $\mathrm{y}$ en el LCR constituyen herramientas muy útiles para el diagnóstico de estos cuadros.

El tratamiento etiológico para Chagas debe iniciarse lo antes posible y debe controlarse la aparición de efectos adversos para realizar tratamiento de los mismos o suspender el fármaco en uso. El HAART debe implementarse en forma sistemática para mejorar los recuentos de linfocitos TCD4+ y evitar nuevas recaídas.

\section{Resumen}

La enfermedad de Chagas, causada por el protozoo Trypanosoma cruzi, es una antropo-zoonosis endémica en Latinoamérica, vinculada con aspectos socio-económicoculturales deficitarios y considerada una de las enfermedades desatendidas. Presentamos un caso fatal de una reactivación de la enfermedad de Chagas con afectación del sistema nervioso central en un paciente con infección por VIH. El diagnóstico se confirmó por reacción de polimerasa en cadena (RPC) positiva en sangre. Tuvo una buena respuesta al tratamiento con benznidazol. Las dificultades en el manejo del tratamiento etiológico se debieron al número limitado de medicamentos antitripanosomiásicos y la aparición de efectos adversos graves.

\section{Referencias bibliográficas}

1.- Enfermedad de Chagas: 100 años después. Boletín de la Organización Mundial de la Salud. 2009. Disponible en: http://www. who.int/bulletin/volumes/87/7/09-030709/es/ (acceso: 17 de noviembre de 2015).

2.- Corti M. AIDS and Chagas' disease. AIDS Patient Care STDS 2000; 14: 581-8.

3.- Souza-Lima R de C, Barbosa M D, Coura J R, Arcanjo A R, Nascimento Ada S, Ferreira J $\mathrm{M}$, et al. Outbreak of acute Chagas disease associated with oral transmission in the Rio Negro region, Brazilian Amazon. Rev Soc Bras Med Trop 2013; 46: 510-4.

4.- Herwaldt B L. Laboratory-acquired parasitic infections from accidental exposures. Clin Microbiol Rev 2001; 14: 659-88.

5.- Oficina regional para las Américas de la Organización mundial de la Salud-Programa de la OPS/OMS Temas de salud: Enfermedad de Chagas 2015. Disponible en: http://www.paho. org $/ \mathrm{hq} / \mathrm{index}$. php?option $=$ com_topics\&view $=$ article\&id $=10 \&$ Itemid $=40743$ (acceso: 17 de noviembre de 2015).

6.- Córdova E, Boschi A, Ambrosioni J, Cudos C, Corti M. Reactivation of Chagas disease with central nervous system involvement in HIV-infected patients in Argentina, 1992-2007. Int J Infect Dis 2008; 12: 587-92.

7.- Pagano M A, Segura M J, Di Lorenzo G A, Garau M L, Molina H A, Cahn P, et al. Cerebral tumor-like American trypanosomiasis in acquired immunodeficiency syndrome. Ann Neurol 1999; 45: 403-6.

8.- Corti M, Trione N, Corbera K, Vivas C.
Chagas disease: another cause of cerebral mass in patients with acquired immunodeficiency syndrome. Enferm Infecc Microbiol Clin 2000; 18: 194-6.

9.- Del Castillo M, Mendoza G, Oviedo J, Pérez-Bianco R, Anselmo A, Silva M. AIDS and Chagas disease with central nervous system tumor-like lesion. Am J Med 1990; 88: 693-4.

10.- Rocha A, de Meneses A C, da Silva A M, Ferreira M S, Nishioka S A, Burgarelli M K, et al. Pathology of patients with Chagas disease and acquired immunodeficiency syndrome. Am J Trop Med Hyg 1994; 50 : 261-8.

11.- Corti M. Enfermedad de Chagas y síndrome de inmunodeficiencia adquirida. Rev Esp Enferm Emerg 2003; 5: 13-7.

12.- Sinagra A, Luna C, Riarte A. Sida y enfermedad de Chagas. Medicina (Bs As) 1999; 59 (supl III): 22.

13.- Madalosso G, Pellini A C, Vasconcelos M J, Ribeiro A F, Weissmann L, Oliveira Filho G S, et al. Chagasic meningoencephalitis: case report of a recently included AIDS-defining illness in Brazil. Rev Inst Med Trop Sao Paulo 2004; 46: 199-202.

14.- Sztokhamer D, Arias L, Dinerstein E, Gustincic M, Gárgano S. Reactivación de enfermedad de Chagas (Tripanosomiasis americana) con compromiso cerebral en pacientes VIH/SIDA en Argentina. Actualizaciones en SIDA 2010; 18: 49-54. http://www.huesped.org.ar/wp-content/ uploads/2014/09/ASEI-68-49-54.pdf (acceso: 17 de noviembre de 2015).

15.- de Almeida E A, Ramos Júnior A N,
Correia D, Shikanai-Yasuda M. Co-infection Trypanosoma cruzi/HIV: systematic review (1980-2010). Rev Soc Bras Med Trop 2011; 44: 762-70.

16.- Iliovich E, López R, Kum M, Usandizaga G. Spontaneous chagasic peritonitis in a patient with AIDS. Medicina (Buenos Aires) 1998; 58: 507-8.

17.- Velásquez J, Oelemann W, Carnevale S, Bessaso H, Etchart C, Cabrera M et al. Trypanosoma cruzi en SIDA: localización gastrointestinal y mecanismos alternativos de transmisión. Rev Hosp Nac Baldomero Sommer 2000; 3: 7-18.

18.- Martínez I, Cervantes-Landín A, Espinoza B. Diagnóstico molecular de la enfermedad de Chagas. Gac Méd Mex 2013; 149: 363-5.

19.- Wincker P, Britto C, Pereira J B, Cardoso M A, Oelemann W, Morel C M. Use of a simplified polymerase chain reaction procedure to detect Trypanosoma cruzi in blood samples from chronic chagasic patients in a rural endemic area. Am J Trop Med Hyg 1994; 51 : 771-7.

20.- Lages-Silva E, Ramírez L, Silva-Vergara M L, Chiari E. Chagasic meningoencephalitis in a patient with acquired immunodeficiency syndrome: diagnosis, follow-up and genetic characterization of Trypanosoma cruzi. Clin Infect Dis 2002; 34: 118-23.

21.- Argentina. Ministerio de Salud de la Nación. Guías para la atención al paciente infectado con Trypanosoma cruzi (Enfermedad de Chagas) 2012 http://www.msal.gob.ar/chagas/ images/stories/Equipos/Guia_Nacional_ Chagas_version_27092012.pdf (acceso: $17 \mathrm{de}$ noviembre de 2015). 
22.- Miller D A, Hernández S, Rodríguez De Armas L, Eelss S J, Traina M M, Miller L G, et al. Tolerance of benznidazole in a United States Chagas disease clinic. Clin Infect Dis 2015; 60 : 1237-40.

23.- Morillo C A, Marín-Neto J A, Avezum A, SosaEstani S, Rassi A Jr, Rosas F, et al. BENEFIT Investigators. Randomized trial of benznidazole for chronic Chagas' cardiomyopathy. N Engl J Med 2015; 373: 1295-306.

24.- Rassi A Jr, Dias J C, Marín-Neto J A, Rassi A. Challenges and opportunities for primary, secondary, and tertiary prevention of Chagas' disease. Heart 2009; 95: 524-34.

25.- Manigot D A. SIDA y Chagas: la dificultad de globalizar los protocolos. Medicina (Buenos Aires) 1998; 58: 522-4.

26.- Organización Panamericana de la salud (OPS). Pautas para la prevención de infecciones oportunistas en personas con VIH o SIDA en América Latina y el Caribe. Washington, DC 2000. http://www.bvsops.org.uy/pdf/vihsida.pdf (acceso: 17 de noviembre de 2015). 